\title{
Exome-wide association studies in general and long-lived populations identify genetic variants related to human age
}

Patrick Sin-Chan ${ }^{1,2}$, Nehal Gosalia ${ }^{1,2}$, Chuan Gao ${ }^{1,2}$, Cristopher V. Van Hout ${ }^{1,2}$, Bin Ye ${ }^{1,2}$, Anthony Marcketta $^{1,2}$, Alexander H. Li ${ }^{1,2}$, Colm O’Dushlaine ${ }^{1,2}$, Dadong Li ${ }^{1,2}$, John D. Overton ${ }^{1,2}$, Jeffrey D. Reid $^{1,2}$, Aris Baras ${ }^{1,2}$, Regeneron Genetics Center ${ }^{1}$, David J. Carey ${ }^{3}$, David H. Ledbetter ${ }^{3}$, Daniel Rader $^{4}$, Marylyn D. Ritchie ${ }^{4}$, Scott M. Damrauer ${ }^{4}$, Sofiya Milman ${ }^{5}$, Nir Barzilai ${ }^{5}$, David J. Glass ${ }^{2}$, Aris N. Economides ${ }^{1,2} \&$ Alan R. Shuldiner ${ }^{1,2 \#}$

${ }^{1}$ Regeneron Genetics Center, Tarrytown, NY, 10591, USA.

${ }^{2}$ Regeneron Pharmaceuticals, Tarrytown, NY, 10591, USA.

${ }^{3}$ Geisinger Health System, Danville, PA, 17822, USA.

${ }^{4}$ Perelman School of Medicine, University of Pennsylvania, Philadelphia, PA, 19104, USA.

${ }^{5}$ Institute for Aging Research, Albert Einstein College of Medicine, Bronx, NY, 10461, USA.

${ }^{*}$ Corresponding author:

Alan R. Shuldiner, MD

777 Old Saw Mill River Road

Tarrytown, New York 20591

Ph: 914-847-1081

Email alan.shuldiner@regeneron.com

Keywords: aging, clonal hematopoiesis of indeterminate potential, exome-wide association studies, founder population, genetics, longevity

\section{SUMMARY}

Aging is characterized by degeneration in cellular and organismal functions leading to increased disease susceptibility and death. Although our understanding of aging biology in model systems has increased dramatically, large-scale sequencing studies to understand human aging are now just beginning. We applied exome sequencing and association analyses (ExWAS) to identify agerelated variants on 58,470 participants of the DiscovEHR cohort. Linear Mixed Model regression analyses of age at last encounter revealed variants in genes known to be linked with clonal hematopoiesis of indeterminate potential, which are associated with myelodysplastic syndromes, as top signals in our analysis, suggestive of age-related somatic mutation accumulation in hematopoietic cells despite patients lacking clinical diagnoses. In addition to APOE, we identified rare DISP2 rs183775254 $\left(\mathrm{p}=7.40 \times 10^{-10}\right)$ and $Z Y G 11 A$ rs74227999 $\left(\mathrm{p}=2.50 \times 10^{-08}\right)$ variants that were negatively associated with age in either both sexes combined and females, respectively, which were replicated with directional consistency in two independent cohorts. Epigenetic mapping showed these variants are located within cell-type-specific enhancers, suggestive of important transcriptional regulatory functions. To discover variants associated with extreme age, 
we performed exome-sequencing on persons of Ashkenazi Jewish descent ascertained for extensive lifespans. Case-Control analyses in 525 Ashkenazi Jews cases (Males $\geq 92$ years, Females $\geq 95$ years) were compared to 482 controls. Our results showed variants in APOE (rs429358, rs6857), and TMTC2 (rs7976168) passed Bonferroni-adjusted p-value, as well as several nominally-associated population-specific variants. Collectively, our Age-ExWAS, the largest performed to date, confirmed and identified previously unreported candidate variants associated with human age.

\section{INTRODUCTION}

Over the past decades, the average human life expectancy has increased dramatically, which has resulted in more people living into adulthood and older ages (WHO, 2015). The number of people in the United States aged 65 years or older is expected to double from 43.1 million in 2012 to $\sim 83.7$ million in 2050 (Ortman et al., 2014). However, increases in life expectancy have not been accompanied by equivalent increases in disease-free lifespan or 'healthspan'. Both the Center for Disease Control and World Health Organization report that the leading causes of death in seniors are cardiovascular diseases, malignancies and neurodegenerative disorders, which suggests aging as the strongest risk factor from developing these age-associated pathologies (Heron, 2019; WHO, 2015). A deeper understanding of aging biology could lead to interventions to decrease the incidence of these age-related diseases, thus increasing healthspan.

There are many factors that may influence human lifespan, which includes diet, gender, ancestry, public health, education, family life, socioeconomic status, social responsibility, access to medical care and genetics. Indeed, several studies have shown a strong familial component to living to older ages. For example, siblings of centenarians exhibit greater chances of living to the ages of their oldest sibling, as compared to the general population (Sebastiani et al., 2016). Moreover, the offspring of long-lived individuals (LLI) are more likely to exhibit delayed onset of age-related diseases and compressed disease morbidity (Atzmon et al., 2004; Dutta et al., 2013; Dutta et al., 2014; Gudmundsson et al., 2000; Lipton et al., 2010; Terry et al., 2003). Multiple reports indicate a genetic component to aging, in which current literature suggests heritability to be between 2035\% (Finch and Tanzi, 1997; Herskind et al., 1996; Sanders et al., 2014; van den Berg et al., 2017), although more recent studies on millions of participants predict lifespan heritability to be much lower (Kaplanis et al., 2018; Timmers et al., 2019). Notably, heritability of lifespan increases at older ages, where effects are most prominent in centenarians and supercentenarians $(>110$ years of age) (Murabito et al., 2012; Perls et al., 2007; Sebastiani and Perls, 2012). The current paradigm is lifespan is a complex polygenic trait, with the inheritance of multiple genes/variants with pleiotropic protective roles across several age-related diseases, and their interaction with environmental and lifestyle factors.

To identify genetic variation associated with human lifespan, research has focused on specific candidate genes and more recently common variant genome-wide association studies (GWAS) (Broer et al., 2015; Deelen et al., 2014; Deelen et al., 2019; Joshi et al., 2017; Pilling et al., 2017; Sebastiani et al., 2017; Zeng et al., 2016). While these past studies have identified multiple genetic variants associated with age, only one variant in apolipoprotein $(A P O E)$ is the most consistently replicated that passes genome-wide significance $\left(\mathrm{p}<5 \times 10^{-08}\right)$ across multiple, independent cohorts 
and in meta-analyses (Partridge et al., 2018). The lack of replication in these studies may be due to small sample size, study-specific age cut-offs to define long-lived status, sex-specific genetic architecture of aging, and genetic and/or lifestyle heterogeneity among cohorts which may compromise meta-analyses. Moreover, the majority of published studies were performed on single candidate genes or common variant genome-wide genotype data, which limits the discovery of rare and novel age-related variants. In this study, we applied exome sequencing and exome-wide association analyses on ages of participants from large general populations and an extreme longlived cohort to identify novel genetic variants associated with human aging.

\section{RESULTS}

\section{Identifying genetic variants that change across strata of ages in the general population}

The Regeneron Genetics Center (RGC) and Geisinger Health System (GHS) established the DiscovEHR study, consisting of GHS patients who participated in the MyCode Community Health Initiative. GHS serves a largely unselected population of $>1.6$ million participants from central Pennsylvania who are of predominantly European ancestry (Abul-Husn et al., 2016; Dewey et al., 2017; Dewey et al., 2016). As a discovery cohort, we analyzed exome sequence data from 58,470 participants of European descent (GHS60K) with a median age of 62 years (range 18-107 years) and median body mass index (BMI) of $30.3 \mathrm{~kg} / \mathrm{m}^{2}$ (range $14.3-57.5 \mathrm{~kg} / \mathrm{m}^{2}$ ) (Figure 1A). GHS60K participants were female $(\mathrm{n}=34,765 ; 59.4 \%)$ and male $(\mathrm{n}=23,705 ; 40.6 \%)$, and majority were alive $(\mathrm{n}=52,653 ; 90.1 \%)$ (Figure 1B-D).

To identify age-associated variants, we performed an 'Age-ExWAS' which entails a linear mixed model (LMM-BOLT) analysis using 'Age at last encounter' obtained from the electronic health record (EHR) as a quantitative trait, as shown in Figure 1B, under an additive model, which was adjusted for four principal components and sex as covariates (Figure 2A). First, as proof-ofconcept, we tested whether 73 previously published candidate age-related variants were also ageassociated in our analysis (Partridge et al., 2018), of which 16 were present in GHS60K exome data (Supplementary Table 1). We replicated APOE (rs429358), APOE (rs2075650) and HFE (rs1800562) variants, which were negatively associated with age and passed the Bonferroni adjusted p-value threshold $\left(\mathrm{p}=3.10 \times 10^{-03}\right)$ (Figure 2B). We next calculated the allelic frequencies of $A P O E$ haplotypes per age group, which revealed the common APOEe4 haplotype, which is associated with Alzheimer's disease and early mortality, decreases in frequency with age $(\mathrm{p}=$ $\left.2.20 \times 10^{-12}\right)$. In contrast, the protective and rarer $A P O E e 2$ haplotype increases in frequency with age $\left(\mathrm{p}=9.90 \times 10^{-08}\right)$ (Figure 2C).

Age-ExWAS revealed variants in genes known to be associated with clonal hematopoiesis of indeterminate potential (CHIP), which exhibited the most significant $p$-values and were positively associated with age in our analysis. These included variants with predicted deleterious functions in ASXL1 (rs756958159), JAK2 (rs77375493), SF3B1 (rs559063155), SRSF2 (rs751713049) and DNMT3A (rs147001633) (Figure 2D-E). CHIP variants are defined as the accumulation of agerelated somatic mutations resulting in clonal expansion of hematopoietic cells in the absence of hematological malignancies clinical phenotypes (Steensma et al., 2015). To confirm whether these suspected CHIP variants are indeed blood-borne somatic mutations that were designated as 
"heterozygotes" with our genotype calling algorithm, we performed a comparative Age-ExWAS on exome sequence data derived from DNA extracted from saliva $(\mathrm{n}=8,102$; median age $=51.6$ years) or a matched sample size from blood $(n=8,102$; median age $=55.2$ years $)$ of the DiscovEHR cohort, which was adjusted for four principal components and sex. Our analysis revealed the five suspected CHIP variants from GHS60K Age-ExWAS analysis were more significantly associated with age from blood samples, in contrast to saliva (Figure 2F). Collectively, this data suggest that these top age-associated variants are likely somatic CHIP variants from blood.

In addition to APOE and suspected CHIP variants, Age-ExWAS in the GHS60K discovery cohort identified 18 additional variants that exceeded genome-wide significance $\left(\mathrm{p}<5 \times 10^{-08}\right)$ (Figure 3A). To validate our findings, we performed Age-ExWAS analysis on two additional replication cohorts, including GHS30K ( $\mathrm{n}=28,930$; median age = 54 years $)$ and UPENN biobank $(\mathrm{n}=8,209$; median age $=68$ years) (Figure 3B; Supplementary Table 2). Interestingly, we observed a rare variant in DISP2 (rs183775254) which was validated with nominal significance and directional consistency in both replication cohorts (Meta-analysis: $\mathrm{p}=2.80 \times 10^{-10}$; Beta $=-12$ years; $\mathrm{MAF}=$ $3.50 \times 10^{-04}$ ) (Figure 3C). While we observed additional variants in/near DISP2, the intronic DISP2 (rs183775254) SNP was the top age-associated variant in our analyses. Gene burden tests did not show any additional associations of DISP2 with age (Supplementary Table 3). This data indicates the specific DISP2 rs183775254 variant, which is located within the first intron of DISP2 and decreases in allele frequency with increasing age, as the top age-related and replicable signal in our analysis.

As this DISP2 variant is negatively associated with age (-12 years in meta-analysis), we next characterized demographics of carriers of this SNP. As expected, the 36 carriers of this variant in GHS60K are younger (median age $=38.8$ years; range $=20-87$ ), as compared to 56,174 noncarriers (median age $=61.4$ years; range $=18-105)$. We performed phenome-wide association studies (Phe-WAS) using GHS and UK Biobank (UKB) phenotype data of participants of European ancestry, which showed no International Statistical Classification of Diseases and Related Health Problems (ICD-10) codes significantly associated with DISP2 rs183775254 (Supplementary Table 4). To explore the clinical significance this SNP, we analyzed electronic health records of 36 carriers of rs 183775254 and age/sex-matched non-carriers to identify ICD-10 codes diagnosed these patients. Interestingly, total burden of ICD-10 codes was significantly higher $\left(\mathrm{p}=4.0 \times 10^{-03}\right)$ in carriers $(2,104$ total; median $=51 \mathrm{ICD}-10$ codes/patient $)$ versus noncarriers (1,253 total; median $=30$ ICD-10 codes/patient $)$, which comprised of 16 disease categories (Supplementary Table 5).

DISP2 is the human homologue of the Dispatched gene in Drosophila, which acts as a transporterlike membrane protein that releases cholesterol-modified sonic hedgehog $(\mathrm{SHH})$ proteins to trigger long-range SHH signaling with roles in early development and embryonic patterning. As the specific DISP2 rs183775254 SNP is intronic, we next sought to map whether this was located in a transcriptional regulatory region by aligning the position of this variant to publicly available regulatory datasets, including DNAse I hypersensitivity, which marks open transcription factoraccessible chromatin, and H3K27Ac chromatin immuno-precipitation sequencing (ChIP-seq) data, which identifies enhancer elements. Notably, we observed this variant is located within an open chromatin region enriched in H3K27Ac marks specific to early human fetal neural stem cells and absent in other cell types, consistent with high DISP2 levels in brain tissue (Supplementary 
Figure 1A-B). Mapping of transcription factor binding sites showed this region is enriched in multiple enhancer-related transcription factors, including MAX, POLR2A, EP300, JUND, RAD21 and CTCF, indicating this variant may be located in a transcriptionally active regulatory hotspot. Further analysis of expression quantitative trait loci (eQTL) data from GTEx in brain tissue did not show associations with DISP2 rs 183775254 . However, four other DISP2 variants were associated with cis eQTL in brain tissue with positive effects on DISP2 expression, which include rs71472433 (brain caudate basal ganglia; $\mathrm{p}=8.99 \times 10^{-28}$; Beta $=0.65$ ), rs56221586 (putamen basal ganglia; $\mathrm{p}=2.17 \times 10^{-24}$; Beta $=0.71$ ), rs12913300 (brain nucleus accumbens basal ganglia; $\mathrm{p}=$ $7.30 \times 10^{-20}$; Beta $\left.=0.52\right)$, and rs56221586 (cortex; $\mathrm{p}=5.88 \times 10^{-11}$; Beta $\left.=0.32\right)$. Collectively, these observations suggest a potential role of DISP 2 and SHH signaling in early brain development and aging.

As multiple studies suggest a gender specific component of aging, we next performed a sexstratified analysis, in which Age-ExWAS analysis was applied to GHS60K females ( $\mathrm{n}=34,756$; median age $=58$ years $)$ and males $(n=23,705$; median age $=65$ years $)$. These analyses revealed 5 and 11 variants that passed genome-wide significance in GHS60K females and males, respectively (Figure 3D; Supplementary Table 2). Variant lookup in sex-stratified GHS30K and UPENN Age-ExWAS analyses showed that CHIP variants, including JAK2, SRSF2, ASXL1, replicated as age-associated variants in both males and females. In addition, we identified an intronic variant in ZYG11A (rs74227999) significantly associated and negatively correlated with age in females, but not in males (Figure 3E). Further meta analyses strengthened this sex-specific associations in females $\left(\mathrm{p}=8.82 \times 10^{-10}\right.$; Beta $=-14.62$ years; MAF $\left.=5.00 \times 10^{-04}\right)$ as compared to males $\left(\mathrm{p}=1.40 \times 10^{-01}\right.$; Beta $\left.=-4.01 ; \mathrm{MAF}=5.00 \times 10^{-04}\right)$. Gene burden test analyses for ZYG11A did not show any significant associations, pointing to ZYG11A (rs74227999) as the top replicable and age-related variant in our sex-stratified analysis (Supplementary Table 3).

We next characterized 51 carriers of the ZYG11A rs74227999 variant in GHS60K, which comprised of 32 female and 19 males. We observed female carriers (median age $=37$ years; range $=20.3-73.1$ ) were younger than male carriers (median age $=59.7$ years; range $=20.9-89.7$ ). PheWAS analysis showed no obvious ICD-10 code association with this variant (Supplementary Table 4). Notably, we observed a significantly higher $\left(\mathrm{p}=2.39 \times 10^{-06}\right)$ burden of diagnosed ICD10 codes in the 51 carriers (4,082 total; median = 54 ICD-10 codes/patient), as compared to 51 age/sex-matched non-carriers (1,983 total; median $=37$ ICD-10 codes/patient), which are classified by disease categories (Supplementary Table 5).

ZYG11A is a member of cell cycle regulators which has roles in driving cellular proliferation. Epigenetic mapping showed the intronic ZYG11A rs74227999 variant is located within a euchromatic region with an active enhancer specific to GM12878, a lymphoblastoid cell line derived from blood of a female donor of European decent and enriched for multiple enhancerrelated transcription factor binding sites. While ZYG11A is highly expressed in testis tissues (Supplementary Figure 2A-B), we did not observe significant association of rs74227999 with eQTL in germinal tissues. However, we observed one variant in ZYG11A rs534070 (p = 4.26x10${ }^{13}$; Beta $=0.29$ ) that was positively associated with cis eQTL is testis. Taken together, these observations indicate rare variants in non-coding regions within DISP2 and ZYGIIA may be 
located within transcriptional regulatory regions, which may affect expression of these or nearby genes.

\section{Exome- and genome-wide association analysis of long-lived case (LLI)-Control cohorts}

There is a general discrepancy in defining the age threshold of long-lived status. Studies calculating the relative 'risk' of siblings living to the ages of their oldest-living sibling showed that the risks increase significantly at extreme ages (> 100 years) (Gudmundsson et al., 2000; Kerber et al., 2001; Sebastiani et al., 2016). These studies also indicate that the difference in 'relative risk' of living to 90 or 95 years is very marginal, which suggests limited heritability of longevity at these ages. Moreover, multiple extreme long-lived individuals are classified as $\geq 95$ years of age, regardless of gender (Ayers et al., 2017; Deluty et al., 2015; Gubbi et al., 2017; Perice et al., 2016). As females generally live to older ages, we calculated and applied a sex-specific age threshold for long-lived status. Based on the 2015 Mortality Data from the National Vital Statistics, Center for Disease Control of Americans of European ancestry, which records the total number and ages of death per 100,000 individuals (Murphy et al., 2017), we defined 95 years as a cutoff for long-lived status in females, which represented $11.8 \%$ of female deaths in 2015 (Figure 4A). In males, $11.8 \%$ of deaths in 2015 occurred at $\geq 93$ years of age. We extended this analysis to include mortality data from 1997-2015, in which we calculated that 95 years for female is equivalent to a median age of 92 years for males (Figure 4B). Thus, in our subsequent analyses, we classified as a longlived-individual (LLI), $\geq 95$ years for females and $\geq 92$ years for males.

We exome sequenced a large collection of LLI of Ashkenazi Jews, a founder population with high degree of endogamy that exhibits longevity phenotypes and decreased incidence of age-related pathologies (Atzmon et al., 2004). Based on our criteria, there were 525 LLI of Ashkenazi Jewish descent (151 males, 374 females) and 482 controls (224 males, 258 females) (Figure 4C), where both the father and mother of the controls passed away before 92 or 95 years of age, respectively. To identify rare and common variants associated with extreme ages, we merged exome sequence data and genome-wide chip genotype data imputed to the 1000 Genomes reference panel and performed Case-Control analyses (SAIGE - refer to methods) using Control and LLI groups as a binary trait, followed by further adjusting for sex as a covariate (Figure 5A). As positive control, we calculated frequencies of $A P O E$ haplotypes in Ashkenazi Jews, which revealed LLIs were enriched for the protective $A P O E e 2$ allele and depleted for the APOEe4 risk allele, as compared to controls (Figure 5B). Moreover, we assessed for association with longevity 28 previously reported candidate variants (Partridge et al., 2018; Singh et al., 2019) and identified variants in APOE (rs6857, rs4420638) and TMTC2 (rs7976168) that passed a Bonferroni-adjusted statistical significance threshold of $\left(\mathrm{p}=1.70 \times 10^{-03}\right)$ (Figure 5C; Supplementary Table 1).

In exome/genome-wide Case-Control analysis in Ashkenazi Jews, the top associated variant for both sexes combined was in STK39 (rs7594207; intronic; $\mathrm{p}=6.55 \times 10^{-07}$; Beta $=0.521$ ). In females only, the top variants were ADAMTS17 (rs73484155; intronic; $\mathrm{p}=5.70 \times 10^{-08}$; Beta $=-0.981$ ) and SP3 (rs 12613192; intergenic; $p=1.66 \times 10^{-07}$; Beta = -1.70) (Figure 5D; Supplementary Table 6). In contrast, the top $\left(\mathrm{p}<1 \times 10^{-05}\right)$ associated variants in male Case-Control analyses was in PRELID2 (rs1982070; intergenic; $\mathrm{p}=6.55 \times 10^{-07}$; Beta $=0.28$ ). Interestingly, of the top signals, 
only the variant in APOEe4 rs429358 passed the Bonferroni adjusted p-value threshold with directional consistency in both Ashkenazi Jew case-control analyses $\left(\mathrm{p}=1.04 \times 10^{-03}\right)$, as well as GHS60K Age-ExWAS analyses $\left(p=2.40 \times 10^{-11}\right)$ (Figure 5E). Taken together, our results suggest both cohort and sex-specific variants associated with extreme ages worthy of follow up in an expanded sample of Ashkenazi Jewish, as well as other extreme long-lived cohorts.

\section{DISCUSSION}

The discovery of new genetic variants associated with human aging is rapidly increasing due to advancement and lowered costs of genome-wide genotyping and next-generation sequencing technologies. In this study, we performed Age-ExWAS analysis on exome sequence data from large general population cohorts. We identified variants in genes involved in age-related clonal hematopoiesis as top age-associated candidates. Somatic variants in CHIP genes are detected in $\sim 1 \%$ of individuals $<50$ years of age, but increase rapidly in frequency with age, where CHIP mutations are detected in $\sim 10 \%$ and $\sim 20 \%$ in persons aged 70 and 90 years of age, respectively (Dorsheimer et al., 2019; Genovese et al., 2014; Jaiswal et al., 2014; Zink et al., 2017). High frequencies of CHIP variants have been reported to be associated with hematologic malignancies as well as atherosclerosis, although whether the latter is independent of age requires further investigation. The most commonly recurrently mutated CHIP genes are epigenetic and chromatin modifying enzymes DNMT3A, ASXL1 and TET2. Indeed, bone-marrow transplant of TET2deficient hematopoietic stem cells was sufficient to accelerate clonal expansion and increase atherosclerotic plaque formation in immunodeficient mouse models, suggesting a causal role of CHIP variants (Fuster et al., 2017). Interestingly, our analyses revealed CHIP variants also present in long-lived persons of Ashkenazi Jewish descent, without history of cancer or cardiovascular diseases (data not shown). Whether total numbers of CHIP variants or a specific combination may act as a biomarker of healthy versus degenerative aging remains to be studied. Additionally, longitudinal studies focused on transcriptional and epigenetic profiling of blood cells with an increasing number of CHIP variants may shed light into the specific cellular states required for CHIP-mediated pathogenesis.

Our analysis identified a rare age-associated variant within DISP2, in which epigenetic mapping revealed to be located within an enhancer specific to primitive neural cells. DISP2 is an upstream effector of SHH signaling with roles in embryonic development and is highly expressed neural tissue (Hall et al., 2019). In the absence of Disp, hedgehog ligands fail to release from donor cells, resulting in impaired early neural and embryonic development (Burke et al., 1999; Ma et al., 2002). In zebrafish, disp 1 transcripts are detected along the skeletal rod and musculature, whereas disp2 is present in the telencephalon and hindbrain (Nakano et al., 2004). In vivo antisense oligonucleotide-mediated silencing of disp1, but not disp2, resulted in disrupted downstream SHH signaling, suggesting alternate downstream mechanisms may be regulated by disp2 (Li et al., 2008). Our studies suggest a potential role of DISP2 in aging, perhaps with relevance to the brain and nervous system, which will require further validation in other populations and functional assessment in animal and cell systems.

We also identified a rare $Z Y G 11 A$ variant to be significantly associated with age in females that is located within an enhancer element in lymphoblastic cells. In C.elegans, zyg11 is implicated in 
meiotic progression and early embryogenesis and exhibits a maternal effect lethal phenotype, in which offspring of homozygous mutant mothers are embryonic lethal (Carter et al., 1990). Intriguingly, ZYG11A is highly expressed in testis, which indicates potential sex-specific role of the ZYG11A rs74227999 variant in females, whereas other variants in this gene may function in male reproductive development. Additional functional validation in sex-stratified models will be needed to test the gender specific roles of $Z Y G 11 A$ variants. High levels of $Z Y G 11 A$ are associated with advanced lung adenocarcinoma and drives cellular proliferation in part via upregulation of cyclin E to promote tumorigenesis (Husni et al., 2019; Wang et al., 2016). Recent studies implicated ZYG11A as downstream of the IGF-1 pathway in p53 wild-type cancer cells (Achlaug et al., 2019). As it is increasingly evident that suppression of IGF signaling is connected to healthy aging and longevity (Harrison et al., 2009; Selman et al., 2009), our data suggests that ZYG11A may play a role in aging, potentially through modulation of IGF signaling.

Consistent with negative associations of these variants with age, analyses of clinical data revealed significantly higher burdens of ICD-10 codes in DISP2 rs183775254 and ZYG11A rs74227999 carriers, as compared to age and sex matched non-carrier controls. Specifically, our data suggests that carriers of these variants exhibit higher diagnoses of ICD-10 codes related to infections, neoplasms, neurodevelopmental disorders and diseases of the vasculature, nervous system, circulatory system, respiratory system, skin and subcutaneous tissue, musculoskeletal system and connective tissues and genitourinary system. The precise biological and genetic mechanisms by which these variants increase disease susceptibility and diagnoses are actively being studied.

Similar with prior longevity studies (Kulminski et al., 2016; Schachter et al., 1994; Sebastiani et al., 2019), our Case-Control association analyses showed association with increased and decreased frequencies of $A P O E e 2$ and $A P O E e 4$ haplotypes, respectively, in LLI as compared to controls. As with many prior GWAS studies (Partridge et al., 2018), our analysis revealed APOE as the only locus that was age-associated and replicated in our studies. Our Case-Control analyses also identified cohort-specific variants suggestively associated with extreme ages. It is important to highlight that while of European ancestry, the Ashkenazi Jews are a founder population with distinct allelic architecture. As the prevalence to living to such extreme long-lived ages is estimated to be $\sim 1 / 10,000$ (Perls et al., 1999), additional efforts will be required to recruit larger cohorts of long-lived individuals for exome profiling to replicate our findings and to identify additional rare longevity-related variants.

In addition to general population regression analyses and case-control analyses in extreme-aged cohorts, an alternative approach to identify aging-related variants is using parental ages of death regressed on offspring genotypes. Recent parental lifespan studies from large populations including the UK Biobank and AncestryDNA genotype data have identified APOE, LPA, $C H R N A 3 / 5, L D L R, S H 2 B 3 / A T X N 2, C D K N 2 B$-AS1 and other study-specific variants as associated with parental ages (Joshi et al., 2017; Pilling et al., 2017; Timmers et al., 2019; Wright et al., 2019). In parallel with our case-control analyses on extreme-aged cohorts, it will be of high interest to extend these analyses to include parental lifespan analyses on exome sequence data to identify rare, coding variants associated with aging.

In summary, our study applied exome sequencing in large patient populations and LLI CaseControl cohorts and identified several candidate gene variants associated with age and/or 
longevity. Further studies will be necessary for further replication and functional validation of genes/variants associated with aging to gain mechanistic insights into aging biology and to inform interventions to promote healthy aging.

\section{AUTHOR CONTRIBUTIONS}

P.S-C and A.R.S conceived the project and wrote the manuscript. All analyses were performed by P.S-C with assistance from N.G, C.G, C.V.V.H, B.Y, A.M, A.H.L, C.O and DL. Exome sequencing and variant calling were performed under the supervision of J.D.O, J.D.R, and A.B. A.E and D.G provided scientific input. DNA for exome sequencing and electronic medical records were generously provided by D.J.C, D.H.L, D.R, M.D.R, S.M.D, S.M and N.B.

\section{CONFLICTS OF INTEREST}

P.S-C, N.G, C.G, C.V.H, B.Y, A.M, A.H.L, C.O, D.L, J.D.O, J.D.R, A.B, D.J.G, A.N.E and A.R.S were employed by Regeneron Pharmaceuticals, and S.M.D supported by the U.S. Department of Veterans Affairs (IK2-CX001780) and received research support from RenalytixAI and personal fee from Calico Labs at the time this study was conducted. 


\section{REFERENCES}

Abul-Husn, N.S., Manickam, K., Jones, L.K., Wright, E.A., Hartzel, D.N., Gonzaga-Jauregui, C., O'Dushlaine, C., Leader, J.B., Lester Kirchner, H., Lindbuchler, D.M., et al. (2016). Genetic identification of familial hypercholesterolemia within a single U.S. health care system. Science 354.

Achlaug, L., Sarfstein, R., Nagaraj, K., Lapkina-Gendler, L., Bruchim, I., Dixit, M., Laron, Z., Yakar, S., and Werner, H. (2019). Identification of ZYG11A as a candidate IGF1-dependent protooncogene in endometrial cancer. Oncotarget 10,4437-4448.

Atzmon, G., Schechter, C., Greiner, W., Davidson, D., Rennert, G., and Barzilai, N. (2004). Clinical phenotype of families with longevity. J Am Geriatr Soc 52, 274-277.

Ayers, E., Barzilai, N., Crandall, J.P., Milman, S., and Verghese, J. (2017). Association of Family History of Exceptional Longevity With Decline in Physical Function in Aging. J Gerontol A Biol Sci Med Sci 72, 1649-1655.

Barzilai, N., Atzmon, G., Schechter, C., Schaefer, E.J., Cupples, A.L., Lipton, R., Cheng, S., and Shuldiner, A.R. (2003). Unique lipoprotein phenotype and genotype associated with exceptional longevity. JAMA 290, 2030-2040.

Broer, L., Buchman, A.S., Deelen, J., Evans, D.S., Faul, J.D., Lunetta, K.L., Sebastiani, P., Smith, J.A., Smith, A.V., Tanaka, T., et al. (2015). GWAS of longevity in CHARGE consortium confirms APOE and FOXO3 candidacy. J Gerontol A Biol Sci Med Sci 70, 110-118.

Burke, R., Nellen, D., Bellotto, M., Hafen, E., Senti, K.A., Dickson, B.J., and Basler, K. (1999). Dispatched, a novel sterol-sensing domain protein dedicated to the release of cholesterol-modified hedgehog from signaling cells. Cell 99, 803-815.

Carter, P.W., Roos, J.M., and Kemphues, K.J. (1990). Molecular analysis of zyg-11, a maternaleffect gene required for early embryogenesis of Caenorhabditis elegans. Mol Gen Genet 221, 7280 .

Deelen, J., Beekman, M., Uh, H.W., Broer, L., Ayers, K.L., Tan, Q., Kamatani, Y., Bennet, A.M., Tamm, R., Trompet, S., et al. (2014). Genome-wide association meta-analysis of human longevity identifies a novel locus conferring survival beyond 90 years of age. Hum Mol Genet 23, 44204432.

Deelen, J., Evans, D.S., Arking, D.E., Tesi, N., Nygaard, M., Liu, X., Wojczynski, M.K., Biggs, M.L., van der Spek, A., Atzmon, G., et al. (2019). A meta-analysis of genome-wide association studies identifies multiple longevity genes. Nat Commun 10, 3669.

Deluty, J.A., Atzmon, G., Crandall, J., Barzilai, N., and Milman, S. (2015). The influence of gender on inheritance of exceptional longevity. Aging (Albany NY) 7, 412-418.

Dewey, F.E., Gusarova, V., Dunbar, R.L., O'Dushlaine, C., Schurmann, C., Gottesman, O., McCarthy, S., Van Hout, C.V., Bruse, S., Dansky, H.M., et al. (2017). Genetic and Pharmacologic Inactivation of ANGPTL3 and Cardiovascular Disease. N Engl J Med 377, 211-221.

Dewey, F.E., Gusarova, V., O'Dushlaine, C., Gottesman, O., Trejos, J., Hunt, C., Van Hout, C.V., Habegger, L., Buckler, D., Lai, K.M., et al. (2016). Inactivating Variants in ANGPTL4 and Risk of Coronary Artery Disease. N Engl J Med 374, 1123-1133.

Dorsheimer, L., Assmus, B., Rasper, T., Ortmann, C.A., Ecke, A., Abou-El-Ardat, K., Schmid, T., Brune, B., Wagner, S., Serve, H., et al. (2019). Association of Mutations Contributing to Clonal Hematopoiesis With Prognosis in Chronic Ischemic Heart Failure. JAMA Cardiol 4, 25-33. 
Dutta, A., Henley, W., Robine, J.M., Langa, K.M., Wallace, R.B., and Melzer, D. (2013). Longer lived parents: protective associations with cancer incidence and overall mortality. J Gerontol A Biol Sci Med Sci 68, 1409-1418.

Dutta, A., Henley, W., Robine, J.M., Llewellyn, D., Langa, K.M., Wallace, R.B., and Melzer, D. (2014). Aging children of long-lived parents experience slower cognitive decline. Alzheimers Dement 10, S315-322.

Finch, C.E., and Tanzi, R.E. (1997). Genetics of aging. Science 278, 407-411.

Fuster, J.J., MacLauchlan, S., Zuriaga, M.A., Polackal, M.N., Ostriker, A.C., Chakraborty, R., Wu, C.L., Sano, S., Muralidharan, S., Rius, C., et al. (2017). Clonal hematopoiesis associated with TET2 deficiency accelerates atherosclerosis development in mice. Science 355, 842-847.

Genovese, G., Kahler, A.K., Handsaker, R.E., Lindberg, J., Rose, S.A., Bakhoum, S.F., Chambert, K., Mick, E., Neale, B.M., Fromer, M., et al. (2014). Clonal hematopoiesis and blood-cancer risk inferred from blood DNA sequence. N Engl J Med 371, 2477-2487.

Gubbi, S., Schwartz, E., Crandall, J., Verghese, J., Holtzer, R., Atzmon, G., Braunstein, R., Barzilai, N., and Milman, S. (2017). Effect of Exceptional Parental Longevity and Lifestyle Factors on Prevalence of Cardiovascular Disease in Offspring. Am J Cardiol 120, 2170-2175.

Gudmundsson, H., Gudbjartsson, D.F., Frigge, M., Gulcher, J.R., and Stefansson, K. (2000). Inheritance of human longevity in Iceland. Eur J Hum Genet 8, 743-749.

Hall, E.T., Cleverdon, E.R., and Ogden, S.K. (2019). Dispatching Sonic Hedgehog: Molecular Mechanisms Controlling Deployment. Trends Cell Biol 29, 385-395.

Harrison, D.E., Strong, R., Sharp, Z.D., Nelson, J.F., Astle, C.M., Flurkey, K., Nadon, N.L., Wilkinson, J.E., Frenkel, K., Carter, C.S., et al. (2009). Rapamycin fed late in life extends lifespan in genetically heterogeneous mice. Nature 460, 392-395.

Heron, M. (2019). Deaths: Leading causes for 2017. National Vital Statistics Reports 68.

Herskind, A.M., McGue, M., Holm, N.V., Sorensen, T.I., Harvald, B., and Vaupel, J.W. (1996). The heritability of human longevity: a population-based study of 2872 Danish twin pairs born 1870-1900. Hum Genet 97, 319-323.

Husni, R.E., Shiba-Ishii, A., Nakagawa, T., Dai, T., Kim, Y., Hong, J., Sakashita, S., Sakamoto, N., Sato, Y., and Noguchi, M. (2019). DNA hypomethylation-related overexpression of SFN, GORASP2 and ZYG11A is a novel prognostic biomarker for early stage lung adenocarcinoma. Oncotarget 10, 1625-1636.

Jaiswal, S., Fontanillas, P., Flannick, J., Manning, A., Grauman, P.V., Mar, B.G., Lindsley, R.C., Mermel, C.H., Burtt, N., Chavez, A., et al. (2014). Age-related clonal hematopoiesis associated with adverse outcomes. N Engl J Med 371, 2488-2498.

Joshi, P.K., Pirastu, N., Kentistou, K.A., Fischer, K., Hofer, E., Schraut, K.E., Clark, D.W., Nutile, T., Barnes, C.L.K., Timmers, P., et al. (2017). Genome-wide meta-analysis associates HLADQA1/DRB1 and LPA and lifestyle factors with human longevity. Nat Commun 8, 910.

Kaplanis, J., Gordon, A., Shor, T., Weissbrod, O., Geiger, D., Wahl, M., Gershovits, M., Markus, B., Sheikh, M., Gymrek, M., et al. (2018). Quantitative analysis of population-scale family trees with millions of relatives. Science 360, 171-175.

Kerber, R.A., O'Brien, E., Smith, K.R., and Cawthon, R.M. (2001). Familial excess longevity in Utah genealogies. J Gerontol A Biol Sci Med Sci 56, B130-139.

Kulminski, A.M., Raghavachari, N., Arbeev, K.G., Culminskaya, I., Arbeeva, L., Wu, D., Ukraintseva, S.V., Christensen, K., and Yashin, A.I. (2016). Protective role of the apolipoprotein E2 allele in age-related disease traits and survival: evidence from the Long Life Family Study. Biogerontology 17, 893-905. 
Li, H., and Durbin, R. (2009). Fast and accurate short read alignment with Burrows-Wheeler transform. Bioinformatics 25, 1754-1760.

Li, N., Flynt, A.S., Kim, H.R., Solnica-Krezel, L., and Patton, J.G. (2008). Dispatched Homolog 2 is targeted by miR-214 through a combination of three weak microRNA recognition sites. Nucleic Acids Res 36, 4277-4285.

Lipton, R.B., Hirsch, J., Katz, M.J., Wang, C., Sanders, A.E., Verghese, J., Barzilai, N., and Derby, C.A. (2010). Exceptional parental longevity associated with lower risk of Alzheimer's disease and memory decline. J Am Geriatr Soc 58, 1043-1049.

Loh, P.R., Tucker, G., Bulik-Sullivan, B.K., Vilhjalmsson, B.J., Finucane, H.K., Salem, R.M., Chasman, D.I., Ridker, P.M., Neale, B.M., Berger, B., et al. (2015). Efficient Bayesian mixedmodel analysis increases association power in large cohorts. Nat Genet 47, 284-290.

Ma, Y., Erkner, A., Gong, R., Yao, S., Taipale, J., Basler, K., and Beachy, P.A. (2002). Hedgehogmediated patterning of the mammalian embryo requires transporter-like function of dispatched. Cell 111, 63-75.

McKenna, A., Hanna, M., Banks, E., Sivachenko, A., Cibulskis, K., Kernytsky, A., Garimella, K., Altshuler, D., Gabriel, S., Daly, M., et al. (2010). The Genome Analysis Toolkit: a MapReduce framework for analyzing next-generation DNA sequencing data. Genome Res 20, 1297-1303.

Murabito, J.M., Yuan, R., and Lunetta, K.L. (2012). The search for longevity and healthy aging genes: insights from epidemiological studies and samples of long-lived individuals. J Gerontol A Biol Sci Med Sci 67, 470-479.

Murphy, S.L., Xu, J.Q., Curtin, C.L., Kochanek, K.D., Curtin, S.C., and Arias, E. (2017). Final data for 2015. National Vital Statistics Reports 66.

Nakano, Y., Kim, H.R., Kawakami, A., Roy, S., Schier, A.F., and Ingham, P.W. (2004). Inactivation of dispatched 1 by the chameleon mutation disrupts Hedgehog signalling in the zebrafish embryo. Dev Biol 269, 381-392.

Ortman, J., Velkoff, V., and Hogan, H. (2014). An Aging Nation: The Older Population in the United States. United States Census Bureau, P25-1140.

Partridge, L., Deelen, J., and Slagboom, P.E. (2018). Facing up to the global challenges of ageing. Nature 561, 45-56.

Perice, L., Barzilai, N., Verghese, J., Weiss, E.F., Holtzer, R., Cohen, P., and Milman, S. (2016). Lower circulating insulin-like growth factor-I is associated with better cognition in females with exceptional longevity without compromise to muscle mass and function. Aging (Albany NY) 8 , 2414-2424.

Perls, T., Kohler, I.V., Andersen, S., Schoenhofen, E., Pennington, J., Young, R., Terry, D., and Elo, I.T. (2007). Survival of parents and siblings of supercentenarians. J Gerontol A Biol Sci Med Sci 62, 1028-1034.

Perls, T.T., Bochen, K., Freeman, M., Alpert, L., and Silver, M.H. (1999). Validity of reported age and centenarian prevalence in New England. Age Ageing 28, 193-197.

Pilling, L.C., Kuo, C.L., Sicinski, K., Tamosauskaite, J., Kuchel, G.A., Harries, L.W., Herd, P., Wallace, R., Ferrucci, L., and Melzer, D. (2017). Human longevity: 25 genetic loci associated in 389,166 UK biobank participants. Aging (Albany NY) 9, 2504-2520.

Reid, J.G., Carroll, A., Veeraraghavan, N., Dahdouli, M., Sundquist, A., English, A., Bainbridge, M., White, S., Salerno, W., Buhay, C., et al. (2014). Launching genomics into the cloud: deployment of Mercury, a next generation sequence analysis pipeline. BMC Bioinformatics 15 , 30 . 
Sanders, J.L., Minster, R.L., Barmada, M.M., Matteini, A.M., Boudreau, R.M., Christensen, K., Mayeux, R., Borecki, I.B., Zhang, Q., Perls, T., et al. (2014). Heritability of and mortality prediction with a longevity phenotype: the healthy aging index. J Gerontol A Biol Sci Med Sci 69, 479-485.

Schachter, F., Faure-Delanef, L., Guenot, F., Rouger, H., Froguel, P., Lesueur-Ginot, L., and Cohen, D. (1994). Genetic associations with human longevity at the APOE and ACE loci. Nat Genet 6, 29-32.

Sebastiani, P., Gurinovich, A., Bae, H., Andersen, S., Malovini, A., Atzmon, G., Villa, F., Kraja, A.T., Ben-Avraham, D., Barzilai, N., et al. (2017). Four Genome-Wide Association Studies Identify New Extreme Longevity Variants. J Gerontol A Biol Sci Med Sci 72, 1453-1464.

Sebastiani, P., Gurinovich, A., Nygaard, M., Sasaki, T., Sweigart, B., Bae, H., Andersen, S.L., Villa, F., Atzmon, G., Christensen, K., et al. (2019). APOE Alleles and Extreme Human Longevity. J Gerontol A Biol Sci Med Sci 74, 44-51.

Sebastiani, P., Nussbaum, L., Andersen, S.L., Black, M.J., and Perls, T.T. (2016). Increasing Sibling Relative Risk of Survival to Older and Older Ages and the Importance of Precise Definitions of "Aging," "Life Span," and "Longevity". J Gerontol A Biol Sci Med Sci 71, 340346.

Sebastiani, P., and Perls, T.T. (2012). The genetics of extreme longevity: lessons from the new England centenarian study. Front Genet 3, 277.

Selman, C., Tullet, J.M., Wieser, D., Irvine, E., Lingard, S.J., Choudhury, A.I., Claret, M., AlQassab, H., Carmignac, D., Ramadani, F., et al. (2009). Ribosomal protein S6 kinase 1 signaling regulates mammalian life span. Science 326, 140-144.

Sin-Chan, P., Mumal, I., Suwal, T., Ho, B., Fan, X., Singh, I., Du, Y., Lu, M., Patel, N., Torchia, J., et al. (2019). A C19MC-LIN28A-MYCN Oncogenic Circuit Driven by Hijacked Superenhancers Is a Distinct Therapeutic Vulnerability in ETMRs: A Lethal Brain Tumor. Cancer Cell 36, 51-67 e57.

Singh, P.P., Demmitt, B.A., Nath, R.D., and Brunet, A. (2019). The Genetics of Aging: A Vertebrate Perspective. Cell 177, 200-220.

Steensma, D.P., Bejar, R., Jaiswal, S., Lindsley, R.C., Sekeres, M.A., Hasserjian, R.P., and Ebert, B.L. (2015). Clonal hematopoiesis of indeterminate potential and its distinction from myelodysplastic syndromes. Blood 126, 9-16.

Terry, D.F., Wilcox, M., McCormick, M.A., Lawler, E., and Perls, T.T. (2003). Cardiovascular advantages among the offspring of centenarians. J Gerontol A Biol Sci Med Sci 58, M425-431.

Timmers, P.R., Mounier, N., Lall, K., Fischer, K., Ning, Z., Feng, X., Bretherick, A.D., Clark, D.W., e, Q.C., Agbessi, M., et al. (2019). Genomics of 1 million parent lifespans implicates novel pathways and common diseases and distinguishes survival chances. Elife 8 .

van den Berg, N., Beekman, M., Smith, K.R., Janssens, A., and Slagboom, P.E. (2017). Historical demography and longevity genetics: Back to the future. Ageing Res Rev 38, 28-39.

Wang, X., Sun, Q., Chen, C., Yin, R., Huang, X., Wang, X., Shi, R., Xu, L., and Ren, B. (2016). ZYG11A serves as an oncogene in non-small cell lung cancer and influences CCNE1 expression. Oncotarget 7, 8029-8042.

WHO (2015). World report on ageing and health. World Health Organization.

Willer, C.J., Li, Y., and Abecasis, G.R. (2010). METAL: fast and efficient meta-analysis of genomewide association scans. Bioinformatics 26, 2190-2191. 
Wright, K.M., Rand, K.A., Kermany, A., Noto, K., Curtis, D., Garrigan, D., Slinkov, D., Dorfman, I., Granka, J.M., Byrnes, J., et al. (2019). A Prospective Analysis of Genetic Variants Associated with Human Lifespan. G3 (Bethesda) 9, 2863-2878.

Zeng, Y., Nie, C., Min, J., Liu, X., Li, M., Chen, H., Xu, H., Wang, M., Ni, T., Li, Y., et al. (2016). Novel loci and pathways significantly associated with longevity. Sci Rep 6, 21243.

Zhou, W., Nielsen, J.B., Fritsche, L.G., Dey, R., Gabrielsen, M.E., Wolford, B.N., LeFaive, J., VandeHaar, P., Gagliano, S.A., Gifford, A., et al. (2018). Efficiently controlling for case-control imbalance and sample relatedness in large-scale genetic association studies. Nat Genet 50, 13351341.

Zink, F., Stacey, S.N., Norddahl, G.L., Frigge, M.L., Magnusson, O.T., Jonsdottir, I., Thorgeirsson, T.E., Sigurdsson, A., Gudjonsson, S.A., Gudmundsson, J., et al. (2017). Clonal hematopoiesis, with and without candidate driver mutations, is common in the elderly. Blood 130, 742-752. 


\section{EXPERIMENTAL PROCEDURES}

\section{Study design and participants}

Age-ExWAS analysis was performed using genomic DNA samples and data from four cohorts, including two DiscovEHR study populations from the MyCode Community Health Initiative of Geisinger Health System (GHS). The GHS discovery cohort (GHS60K) consisted of 58,470 persons of European ancestry who were recruited from outpatient primary care and specialty clinics. Replication cohorts consisted of 28,930 additional persons of European ancestry from the DiscovEHR study (GHS30K) and 8,209 persons of European ancestry from the University of Pennsylvania (UPENN) Biobank. Our case-control analyses consisted of 1,007 persons of Ashkenazi Jewish descent, recruited by Albert Einstein College of Medicine (Barzilai et al., 2003).

\section{DNA Sample Preparation and sequencing}

DNA sample preparation and exome sequencing for participants were performed at the Regeneron Genetics Center, as previously described (Abul-Husn et al., 2016; Dewey et al., 2017; Dewey et al., 2016). Briefly, exome capture was performed using a custom reagent kit from Kapa Biosystems using a fully-automated approach developed at the Regeneron Genetics Center. A unique 6 base pair barcode was added to each DNA fragment during library preparation to facilitate multiplexed exome capture and sequencing. Equal amounts of sample were pooled prior to exome capture with NimbleGen probes on SeqCap VCRome or IDT xGen platforms. Multiplexed samples were sequenced using 75 bp paired-end sequencing on an Illumina v4 HiSeq 2500 to a coverage depth sufficient to provide greater than 20x haploid read depth. Raw sequence data from each Illumina Hiseq 2500 run were uploaded to DNAnexus (Reid et al., 2014) for sequence read alignment and variant identification. Raw sequence data were converted from BCL files to samplespecific FASTQ-files, which were aligned to the human reference build GRCh37.p13 with BWAmem (Li and Durbin, 2009). Single nucleotide variants (SNV) and insertion/deletion (INDEL) sequence variants were identified using the Genome Analysis Toolkit (McKenna et al., 2010).

\section{Exome-wide association study and analytical quality control}

We utilized BOLT-LMM v2.2 to test for associations between variants and age for DiscovEHR and UPENN exome sequence data, which uses the mixed models of association approach (Loh et al., 2015). Genetic relatedness matrix (GRM), which captures population structure from ancestry and relatedness, was included as a random-effects covariate. The GRM was constructed from 39,858 non-MHC markers, with no greater than 1\% genotype missingness, and with minor allele frequency $>0.1 \%$. Logistic regression analyses adjusted for sex and the first four principal components of ancestry. Individual study results were meta-analyzed using METAL (Willer et al., 2010) on plink 1.9. Case-control analyses on Ashkenazi Jewish cohorts were performed using Scalable and Accurate Implementation of GEneralized mixed model (SAIGE) to control for sample relatedness (Zhou et al., 2018), and adjusted for sex as covariate (except in analyses stratified by sex). Variants were filtered using a Minor Allele Count of $\geq 5$. Following this, SNPs were filtered using Quality Depth of $\geq 3$, Read Depth of $\geq 7$ and Allele Balance of $\geq 15 / 85$, whereas INDEL were filtered using Quality Depth of $\geq 5$, Read Depth of $\geq 10$ and Allele Balance of $\geq$ 20/80. 


\section{Imputation}

Ashkenazi Jewish genome-wide genotype data (Illumina GSA array) of European participants were converted to hg19 format, duplicate SNPs removed, and quality controlled by excluding variants with high missingness (> 10\%), high deviations from Hardy-Weinberg equilibrium $\left(1.00 \times 10^{-15}\right)$ and minor allele frequency $<1 \%$. Variants were imputed to HRC-1000G reference panel using Michigan Imputation Server. Imputed variants with info score $>0.3$ were kept, liftedover to hg38 format and merged with exome sequence data. For overlapping variants, those from exome data were selected.

\section{Epigenetic mapping of regulatory regions}

DNase I hypersensitive site, histone marks and transcription factor binding sites were downloaded from ENCODE cell line public data. H3K27Ac ChIP-seq data on human neural stem cell was downloaded from previously published studies (Sin-Chan et al., 2019).

\section{Calculation of $A P O E$ haplotype copies}

To calculate number of copies of $A P O E$ haplotypes in long-lived cohorts, the rs 429358 (Cys130Arg) and rs7412 (Arg176Cys) variants were extracted from exome sequence data. Based on the specific combinations, we determined the APOE haplotypes: $e 2$ (Cys130/Cys176), e3 (Cys130/Arg176) and $e 4$ (Arg130/Arg176). We restricted our analysis on individuals with $e 2 / e 2$, $e 2 / e 3, e 3 / e 3, e 3 / e 4$ and $e 4 / e 4$ genotypes, resulting in 475 controls and 517 LLI of Ashkenazi Jewish descent. The p-value, odds ratio and confidence intervals were calculated using logistic regression, which was normalized for Sex as covariate. 


\section{FIGURE LEGENDS}

\section{Figure 1: Demographics of DiscovEHR cohort}

A) Table of median, ranges and standard deviation of age, body mass index, height and weight of 58,470 participants (GHS60K) of European descent from the DiscovEHR study. B) Age distribution of GHS60K; number of participants in bins 18-20 years and > 80years of age. C-D) Distribution of male/female and alive/deceased status for GHS60K participants.

\section{Figure 2: $A P O E$ and CHIP variants are top age-associated hits in Age-ExWAS}

A) Linear mixed model (LMM-BOLT) analysis on exome sequence data using 'Age at last encounter' as the trait of interest under the additive model and normalized for sex and 4 principal components, followed by LD clumping $\left(\mathrm{r}^{2}<0.1\right)$. Analytical quality control was performed using criteria defined in methods. B) Forest plot of reported age-related variants from published literature. Variant name, N, Beta (non-transformed), SE, MAF and p-value are shown. X-axis shows Beta in years. C) Allele frequencies of carriers of APOEe 2 and APOEe 4 haplotypes plotted based on age deciles. D) Manhattan plot of Age-ExWAS $(\lambda=1.1)$ in GHS60K. Associations $\log 10$ (p-value) for each genome-wide SNP (y-axis) by chromosomal position (x-axis). Red line indicates the threshold for genome-wide statistical significance $\left(\mathrm{p}=5 \times 10^{-08}\right)$. Variants reported as clonal hematopoiesis of indeterminate potential (CHIP) and APOE are shown in black and blue, respectively. E) Table of top 5 suspected CHIP variants where RSID, gene name, prediction function, Beta (non-transformed), p-value and MAF. F) Age-ExWAS on exome sequence data of DNA extracted from 8,102 blood or 8,102 saliva samples. The top suspected CHIP variants are shown in the scatterplot where - $\log 10$ (p-value) of Blood Age-ExWAS (x-axis) and Saliva (AgeExWAS) y-axis. $\mathrm{SE}=$ standard error; $\mathrm{MAF}=$ minor allele frequency.

\section{Figure 3: DISP2 and $Z Y G 11 A$ as age-associated variants in general populations}

A) Table of age-related variants from GHS60K Age-ExWAS analysis that passed genome-wide significance $\left(\mathrm{p}<5 \times 10^{-08}\right)$. RSID, gene name, predicted function, untransformed Beta, $\mathrm{p}$-value, MAF and number of carriers are shown. B) Demographics of replication cohorts of European descent including 28,930 additional participants from GHS30K and 8,209 participants from UPENN Biobank. Total N, median age and male-to-female ratio are shown. C) Forrest plot analysis showing Age-ExWAS results of DISP2 variant in GHS60K, GHS30K and UPENN and meta-analysis. Non-transformed Beta and standard error (SE) in years, direction and p-values for each cohort are shown. D) Manhattan plot of female-only $(\lambda=1.07)$ and male-only $(\lambda=1.05)$ Age-ExWAS in GHS60K. Associations - $\log 10$ (p-value) for each genome-wide SNP (y-axis) by chromosomal position (x-axis) are shown. Red line indicates the threshold for genome-wide statistical significance $\left(\mathrm{p}=5 \times 10^{-08}\right)$. Top variants that passed genome-wide-significance are labeled. E) Forrest plots of ZYG11A in female and male GHS60K, GHS30K, UPENN and metaanalysis. $\mathrm{SE}=$ standard error; $\mathrm{MAF}=$ minor allele frequency.

\section{Figure 4: Determination of sex-specific age threshold of long-lived status}

A) Distribution of total number and ages of deaths per 100,000 individuals of European descent in the United States in 2015. B) Mortality data (1997-2015) showing 95 years in female is equivalent to 92 years in males. C) Table showing total $\mathrm{N}$, median ages and sex distribution of controls and long-lived individuals (LLI) of Ashkenazi Jewish descent. 
Figure 5: Case-control analyses in Ashkenazi Jews long-lived cohort

A) Case-Control analysis using SAIGE was performed on exome sequence data merged with genotype data imputed to 1000 Genome reference panel using Control and LLI groups as a binary trait, followed by further adjusting for sex as covariate, followed by LD clumping $\left(\mathrm{r}^{2}<0.1\right)$. Analytical quality control was performed using criteria defined in methods. B) Table showing frequencies, odd ratio (OR) and p-values of APOEe2-4 haplotypes in LLIs and controls. Percentage of individuals carrying the given $A P O E$ haplotype copies shown in red. C) Of 28 published positive control SNPs (Supplementary Table 1), variants that passed Bonferroni adjusted p-values $\left(\mathrm{p}=1.70 \times 10^{-03}\right)$ are highlighted in yellow. D) Manhattan plots for Ashkenazi Jew cohort both sexes combined, female-only and male-only showing associations of - $\log 10$ (p-value) for each genome-wide SNP (y-axis) by chromosomal position (x-axis). Red line indicates the threshold for genome-wide statistical significance $\left(\mathrm{p}=5 \times 10^{-08}\right)$. Top-age-associated variants are labelled with nearest gene. QQ plots depicting minor genomic inflations are shown. E) Forest plot shows variant in APOE rs429358 in both sexes, female and males only in Ashkenazi Jews (top) and GHS60K Age-ExWAS (bottom). OR = odds ratio; $\mathrm{SE}=$ standard error; $\mathrm{MAF}=$ minor allele frequency.

\section{SUPPLEMENTARY FIGURE LEGENDS}

\section{Supplementary Figure 1: Enhancer mapping and tissue expression of DISP2}

A) Schematic map of DISP2 (chr15:40,649,698-40,652,202) relative to UCSC hg19 RefSeq annotation and ENCODE tracks. Variant of interest is highlighted in the red box and shown relative to ENCODE DNAse I hypersensitive sites, H3K27Ac-ChIP-seq from fetal human neural stem cells and ENCODE cell lines and ENCODE ChIP-seq map of enhancer-related transcription factor binding sites. B) Expression levels of DISP2 from Genotype-Tissue Expression (GTEx) database.

\section{Supplementary Figure 2: Enhancer mapping and tissue expression of ZYG11A}

A) Schematic map of ZYG11A (chr1:53,310,672-53,335,672) relative to UCSC hg19 RefSeq annotation and ENCODE tracks. Variant of interest is highlighted in the red box and shown relative to ENCODE DNAse I hypersensitive sites, H3K27Ac-ChIP-seq from fetal human neural stem cells and ENCODE cell lines and ENCODE ChIP-seq map of enhancer-related transcription factor binding sites. B) Expression levels of ZYG11A from Genotype-Tissue Expression (GTEx) database.

\section{SUPPLEMENTARY TABLES}

Table 1 - Positive control variants and lookup in analyses

Table 2 - Genome-wide significant variants from GHS60K Age-ExWAS analysis and lookup replication cohorts

Table 3 - DISP2 and ZYG11A variants and Burden test results in GHS60K Age-ExWAS

Table 4 - Phenome-wide association study (Phe-WAS) analysis of age-related variants 
Table 5 - Distribution of disease traits in DISP2 and ZYG11A carriers

Table 6 - Ashkenazi Jew Case-Control top hits $\left(\mathrm{p}<1 \times 10^{-05}\right)$ and lookup in GHS60K Age-ExWAS analyses 
Figure 1: Demographics of DiscovEHR cohort

\begin{tabular}{cccc} 
A Demographics of & $\mathbf{5 8 , 4 7 0}$ DiscovEHR participants of European descent \\
\cline { 2 - 5 } Trait & Median & Range & Standard Deviation \\
\hline Median Age, years & 62 & $18-107$ & 17.2 \\
Body Mass index, $\mathrm{kg} / \mathrm{m}^{2}$ & 30.3 & $14.3-57.5$ & 7.34 \\
Height, $\mathrm{cm}$ & 167.6 & $91.4-210.8$ & 10 \\
Weight, $\mathrm{kg}$ & 85.9 & $3.4-254.9$ & 40.7 \\
\hline
\end{tabular}

B

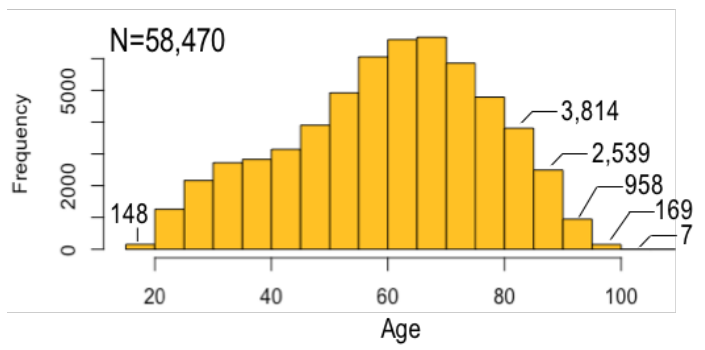

C

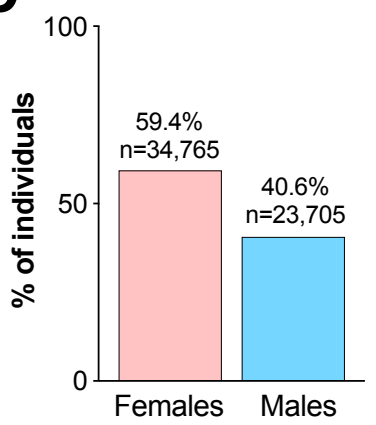

D

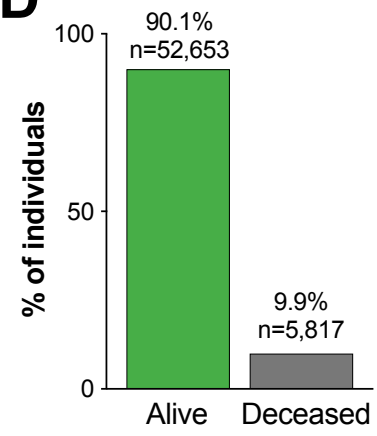


Figure 2: $A P O E$ and CHIP variants are top age-associated hits in Age-ExWAS

A

Whole exomes of 58,470 participants of European descent from DiscovEHR

LMM-BOLT using 'Age at last encounter' as quantitative trait

(Adjust for 4PCs and Sex)

LD clump $\left(r^{2}<0.1\right)$

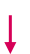

Analytical quality control and data mining
B Previously reported age-related variants in GHS60K Age-ExWAS

\begin{tabular}{|c|c|c|c|c|c|c|}
\hline Variant & $\mathrm{N}$ & Beta (years) & SE & MAF & & $p$-value \\
\hline APOE (rs429358) & 58151 & -0.987 & 0.151 & 0.130 & $\longmapsto$ & $2.40 \mathrm{E}-11$ \\
\hline APOE (rs2075650) & 58354 & -0.534 & 0.146 & 0.138 & $\longmapsto$ & $5.20 \mathrm{E}-05$ \\
\hline HFE (rs 1800562) & 58354 & -0.571 & 0.215 & 0.058 & : & $9.40 \mathrm{E}-03$ \\
\hline USP42 (rs3764814) & 58344 & -0.408 & 0.191 & 0.076 & & $3.00 \mathrm{E}-02$ \\
\hline SIRT1 (rs2273773) & 58351 & 0.351 & 0.202 & 0.067 & & $5.50 \mathrm{E}-02$ \\
\hline SH2B3/ATXN2 (rs3184504) & 58354 & 0.104 & 0.101 & 0.494 & 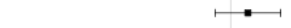 & $1.10 \mathrm{E}-01$ \\
\hline $\mathrm{KL}$ (rs9536314) & 58354 & 0.028 & 0.143 & 0.146 & $\longrightarrow$ & 3.50E-01 \\
\hline KL (rs9527026) & 58354 & 0.028 & 0.143 & 0.146 & $\longmapsto$ & 3.50E-01 \\
\hline SIRT3 (rs11555236) & 58341 & 0.068 & 0.121 & 0.224 & $\longmapsto$ & $3.90 \mathrm{E}-01$ \\
\hline FURIN/FES (rs6224) & 57896 & 0.094 & 0.101 & 0.475 & $\longrightarrow$ & $4.60 \mathrm{E}-01$ \\
\hline CETP (rs5882) & 58277 & 0.117 & 0.109 & 0.687 & $=$ & $4.80 \mathrm{E}-01$ \\
\hline TP53 (rs 1042522) & 58335 & -0.090 & 0.116 & 0.746 & $\longmapsto$ & $5.40 \mathrm{E}-01$ \\
\hline GPX1 (rs1050450) & 58354 & 0.032 & 0.111 & 0.294 & $\longmapsto$ & $6.00 \mathrm{E}-01$ \\
\hline PON1 (rs662) & 58353 & -0.078 & 0.112 & 0.283 & $\longmapsto$ & $6.90 \mathrm{E}-01$ \\
\hline IGF1R (rs2229765) & 58354 & 0.046 & 0.101 & 0.447 & $\longmapsto$ & $8.60 \mathrm{E}-01$ \\
\hline
\end{tabular}

C APOE haplotypes

$\varepsilon 4$

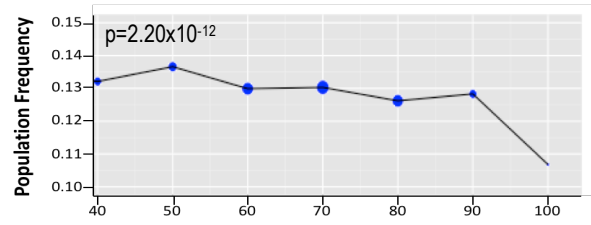

$\varepsilon 2$
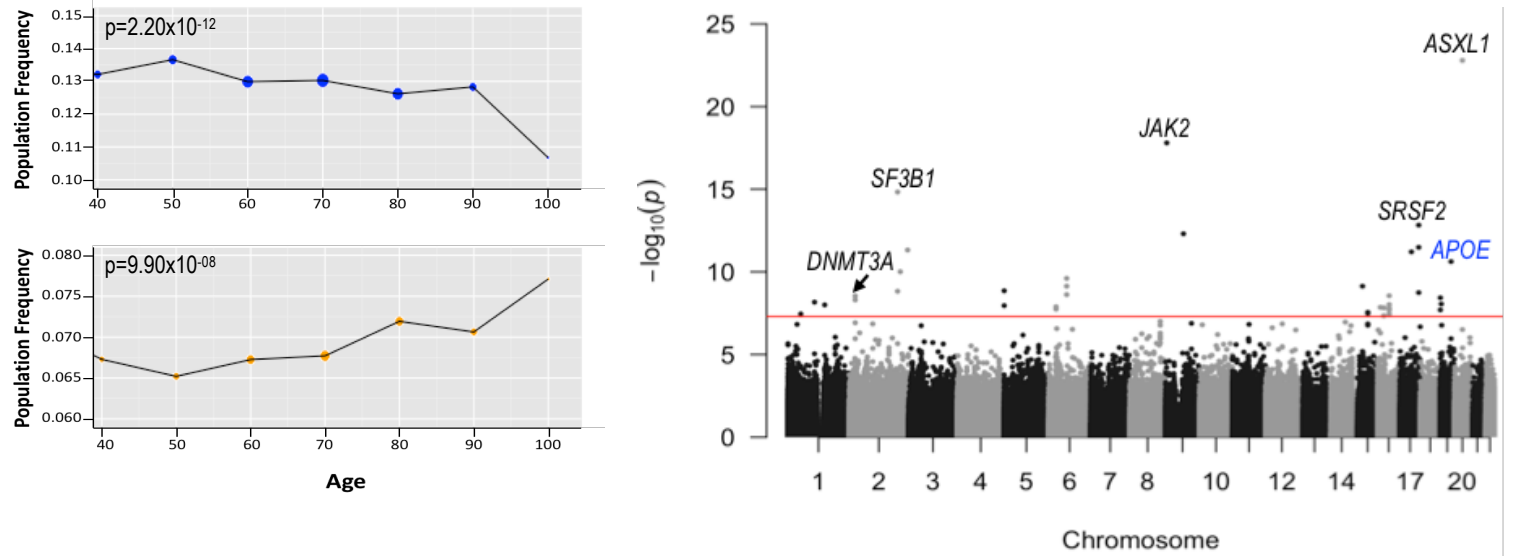

E

Suspected variants in CHIP genes in Blood samples

\begin{tabular}{cccccc}
\hline RSID & Gene & Function & $\begin{array}{c}\text { Beta } \\
\text { (years) }\end{array}$ & p-value & Frequency \\
\hline rs756958159 & ASXL1 & frameshift & 13.70 & $1.60 \mathrm{E}-23$ & $1.40 \mathrm{E}-03$ \\
rs77375493 & JAK2 & missense & 12.00 & $1.60 \mathrm{E}-18$ & $8.32 \mathrm{E}-04$ \\
rs559063155 & SF3B1 & missense & 19.80 & $1.50 \mathrm{E}-15$ & $3.94 \mathrm{E}-04$ \\
rs751713049 & SRSF2 & missense & 21.70 & $3.30 \mathrm{E}-12$ & $3.01 \mathrm{E}-04$ \\
rs147001633 & DNMT3A & missense & 13.20 & $2.90 \mathrm{E}-09$ & $5.14 \mathrm{E}-04$ \\
\hline
\end{tabular}

F

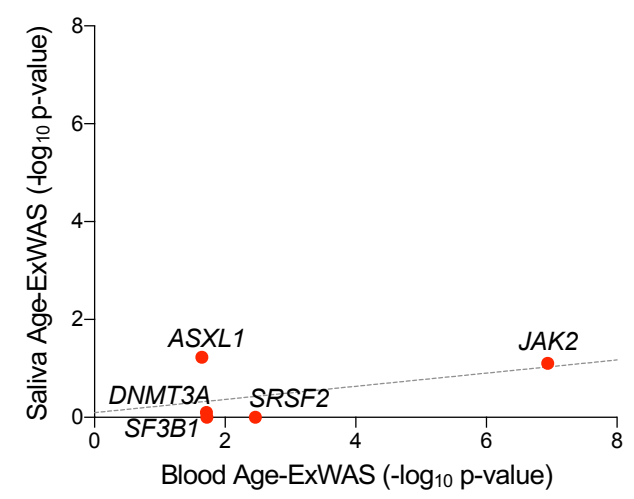


bioRxiv preprint doi: https://doi.org/10.1101/2020.07.19.188789; this version posted July 19, 2020. The copyright holder for this preprint (which was not certified by peer review) is the author/funder. All rights reserved. No reuse allowed without permission.

Figure 3: DISP2 and ZYG11A as age-associated variants in general populations

\begin{tabular}{ccccccc} 
A Genome-wide significant variants & from GHS60K Age-ExWAS & \\
\cline { 2 - 7 } RSID & Variant & Function & $\begin{array}{c}\text { Beta } \\
\text { (years) }\end{array}$ & p-value & MAF & $\begin{array}{c}\text { Number of subjects } \\
\text { Ref/Ref|Ref/Alt|Alt/Alt }\end{array}$ \\
\hline rs955075371 & TMC1 & intronic & 2.53 & $5.00 \times 10^{-13}$ & $3.78 \times 10^{-02}$ & $37479|2990| 36$ \\
rs367917223 & SCLY & intronic & 1.44 & $4.80 \times 10^{-12}$ & $1.20 \times 10^{-01}$ & $25522|6884| 518$ \\
rs765851979 & CDC27 & intronic & 0.78 & $6.20 \times 10^{-12}$ & $3.46 \times 10^{-01}$ & $21162|22794| 5801$ \\
rs429358 & APOE & missense & -0.99 & $2.40 \times 10^{-11}$ & $1.30 \times 10^{-01}$ & $43924|13299| 928$ \\
rs941059731 & PIKFYVE & intronic & 1.17 & $9.80 \times 10^{-11}$ & $1.03 \times 10^{-01}$ & $38214|8883| 451$ \\
NA & KCNQ5 & intronic & 16.67 & $2.50 \times 10^{-10}$ & $3.34 \times 10^{-04}$ & $58304|30| 0$ \\
rs183775254 & DISP2 & intronic & $-\mathbf{1 8 . 3 1}$ & $\mathbf{7 . 4 0 \times 1 0 ^ { - 1 0 }}$ & $\mathbf{3 . 0 9 \times 1 0 ^ { - 0 4 }}$ & $\mathbf{5 8 2 6 8 | 3 6 | 0}$ \\
NA & AHRR & frameshift & -11.33 & $1.40 \times 10^{-09}$ & $6.00 \times 10^{-04}$ & $58271|70| 0$ \\
rs769293627 & GPT2 & missense & 22.83 & $2.80 \times 10^{-09}$ & $1.46 \times 10^{-04}$ & $58315|17| 0$ \\
NA & NDUFS7 & missense & -23.89 & $3.70 \times 10^{-09}$ & $1.37 \times 10^{-04}$ & $58337|16| 0$ \\
rs372276219 & STXBP3 & intronic & 2.20 & $6.80 \times 10^{-09}$ & $2.29 \times 10^{-02}$ & $48312|2323| 0$ \\
NA & KDM4B & intronic & -2.00 & $8.80 \times 10^{-09}$ & $2.18 \times 10^{-02}$ & $46539|2119| 1$ \\
rs148013046 & ANP32E & intronic & 1.07 & $1.00 \times 10^{-08}$ & $9.15 \times 10^{-02}$ & $43572|8759| 451$ \\
NA & AHRR & frameshift & -10.95 & $1.10 \times 10^{-08}$ & $5.91 \times 10^{-04}$ & $58264|69| 0$ \\
rs978403206 & TRIM10 & downstream & 18.96 & $1.30 \times 10^{-08}$ & $2.31 \times 10^{-04}$ & $58321|27| 0$ \\
NA & CLEC16A & intronic & 12.97 & $1.40 \times 10^{-08}$ & $4.88 \times 10^{-04}$ & $58287|57| 0$ \\
rs769305227 & SLC5A11 & intronic & 20.90 & $1.50 \times 10^{-08}$ & $1.80 \times 10^{-04}$ & $58330|21| 0$ \\
NA & VPS13C & intronic & 13.83 & $2.80 \times 10^{-08}$ & $4.15 \times 10^{-04}$ & $57796|48| 0$ \\
rs74227999 & ZYG11A & intronic & -13.79 & $\mathbf{3 . 5 0 \times 1 0 ^ { - 0 8 }}$ & $\mathbf{4 . 3 7 \times 1 0 ^ { - 0 4 }}$ & $\mathbf{5 8 2 9 6 | 5 1 | 0}$ \\
\hline
\end{tabular}
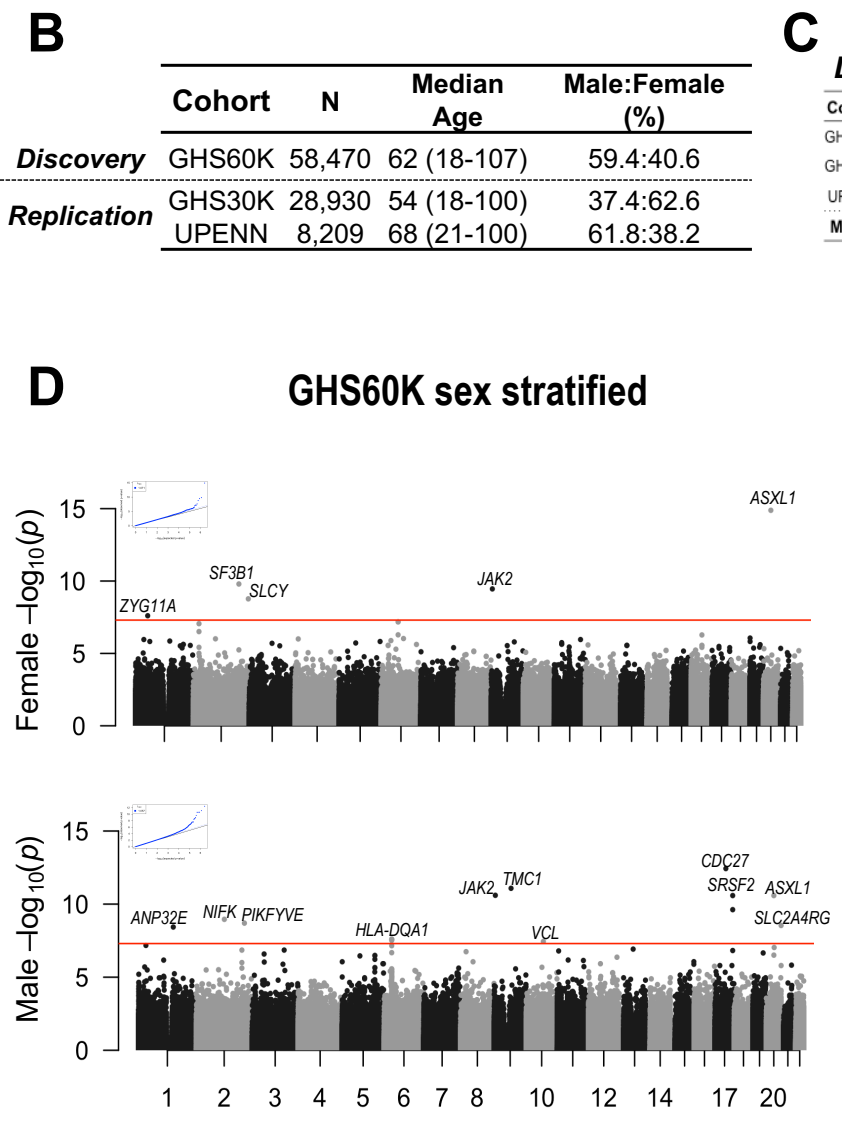

Chromosome
C

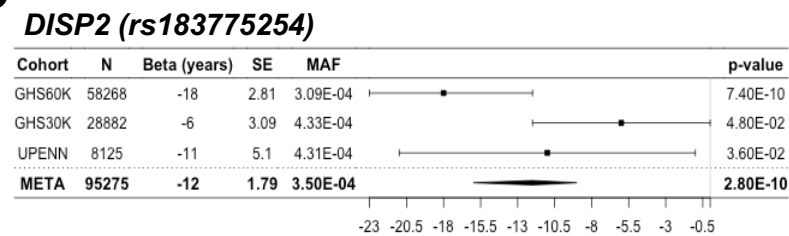

E

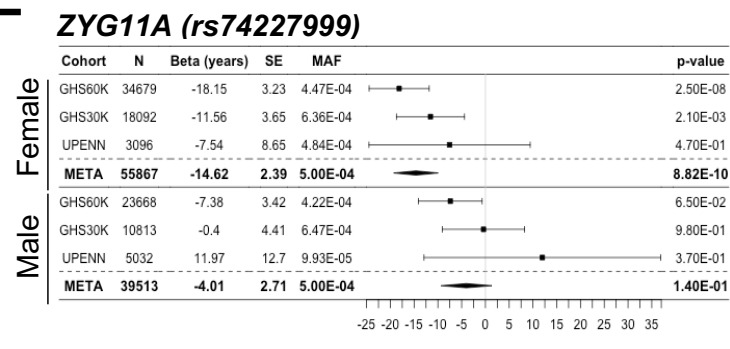


Figure 4: Determination of sex-specific age threshold of long-lived status

A Number of deaths in United States (2015)

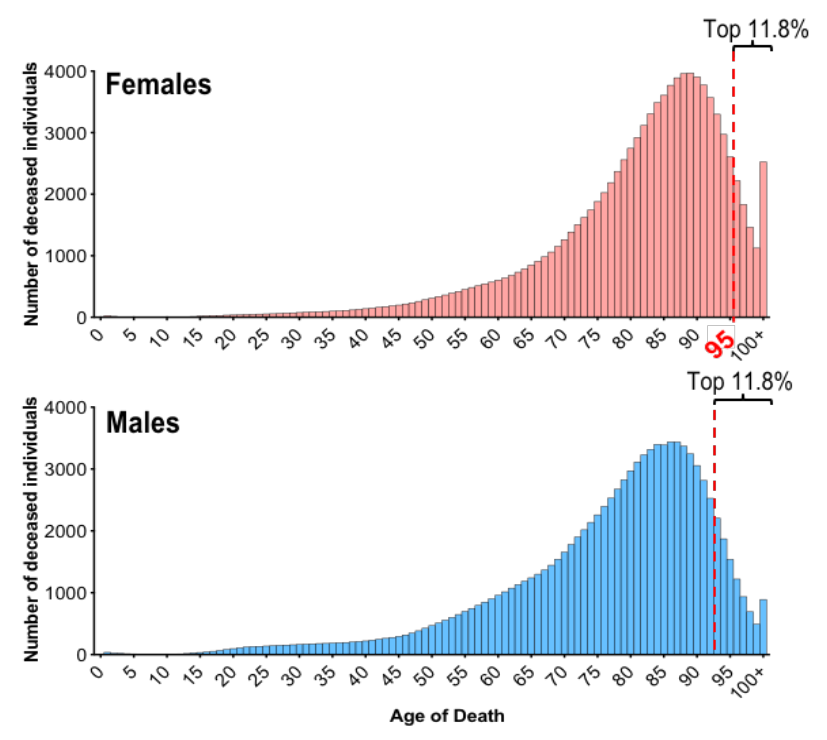

B

\begin{tabular}{cccc}
\hline Year & $\begin{array}{c}\text { Number of females living } \\
\mathbf{2} \mathbf{9 5} \text { years }(\mathbf{1 0 0 , 0 0 0})\end{array}$ & $\begin{array}{c}\text { \% of Females living } \\
\mathbf{2} \mathbf{9 5} \text { years }\end{array}$ & $\begin{array}{c}\text { Equivalent \% in Male } \\
\text { years of life }\end{array}$ \\
\hline 2015 & 11,794 & 11.79 & 93 \\
2014 & 12,332 & 12.33 & 93 \\
2013 & 11,891 & 11.89 & 93 \\
2012 & 11,910 & 11.91 & 93 \\
2011 & 11,656 & 11.66 & 93 \\
2010 & 11,810 & 11.81 & 92 \\
2009 & 12,047 & 12.05 & 92 \\
2008 & 10,934 & 10.93 & 92 \\
2007 & 10,916 & 10.92 & 92 \\
2006 & 10,656 & 10.66 & 92 \\
2005 & 9,924 & 9.92 & 92 \\
2004 & 12,362 & 12.36 & 91 \\
2003 & 11,249 & 11.25 & 92 \\
2002 & 10,571 & 10.57 & 91 \\
2001 & 10,451 & 10.45 & 91 \\
2000 & 9,638 & 9.64 & 91 \\
1999 & 9,541 & 9.54 & 91 \\
1998 & 9,906 & 9.91 & 91 \\
1997 & 9,603 & 9.60 & 91 \\
\hline \multicolumn{5}{r}{ The equivalent to Female age 95 in Male is: } \\
\hline
\end{tabular}

C

\begin{tabular}{cccc}
\cline { 2 - 4 } & \multicolumn{3}{c}{ Ashkenazi Jews } \\
\cline { 2 - 4 } Control & Total & Males & Females \\
\hdashline LLI & 482 & 224 & 258 \\
\cline { 2 - 4 } & 525 & 151 & 374 \\
\hline
\end{tabular}


bioRxiv preprint doi: https://doi.org/10.1101/2020.07.19.188789; this version posted July 19, 2020. The copyright holder for this preprint (which was not certified by peer review) is the author/funder. All rights reserved. No reuse allowed without permission.

Figure 5: Case-control analyses in Ashkenazi Jews long-lived cohort

A

Whole exome sequence merged with imputed data

SAIGE using 'Control' and 'LLI' groups as binary trait

(Adjust for Sex)

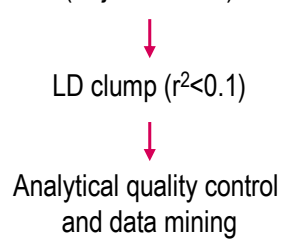

D
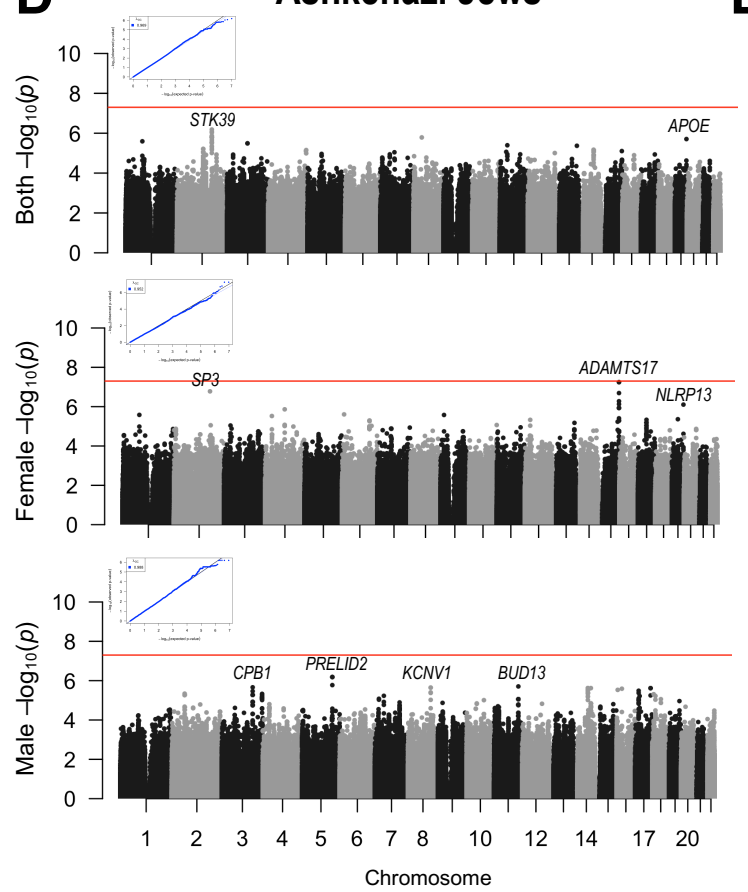

E

\section{APOE (rs429358)}

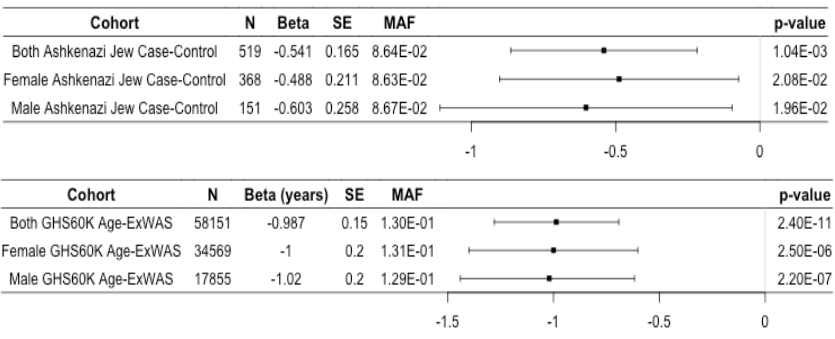


bioRxiv preprint doi: https://doi.org/10.1101/2020.07.19.188789; this version posted July 19, 2020. The copyright holder for this preprint (which was not certified by peer review) is the author/funder. All rights reserved. No reuse allowed without permission.

\section{Supplementary Figure 1: Enhancer mapping and tissue expression of DISP2}

A

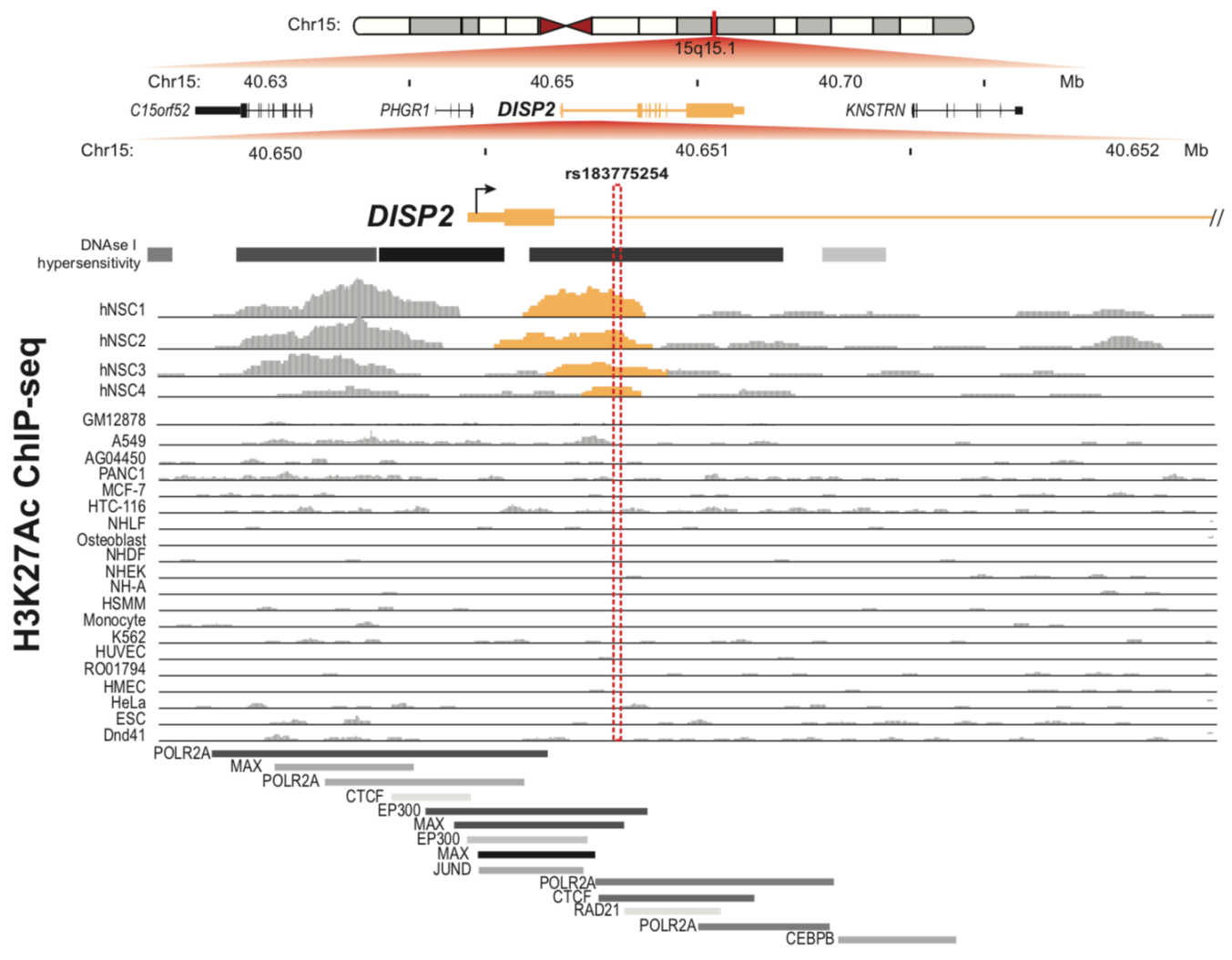

B

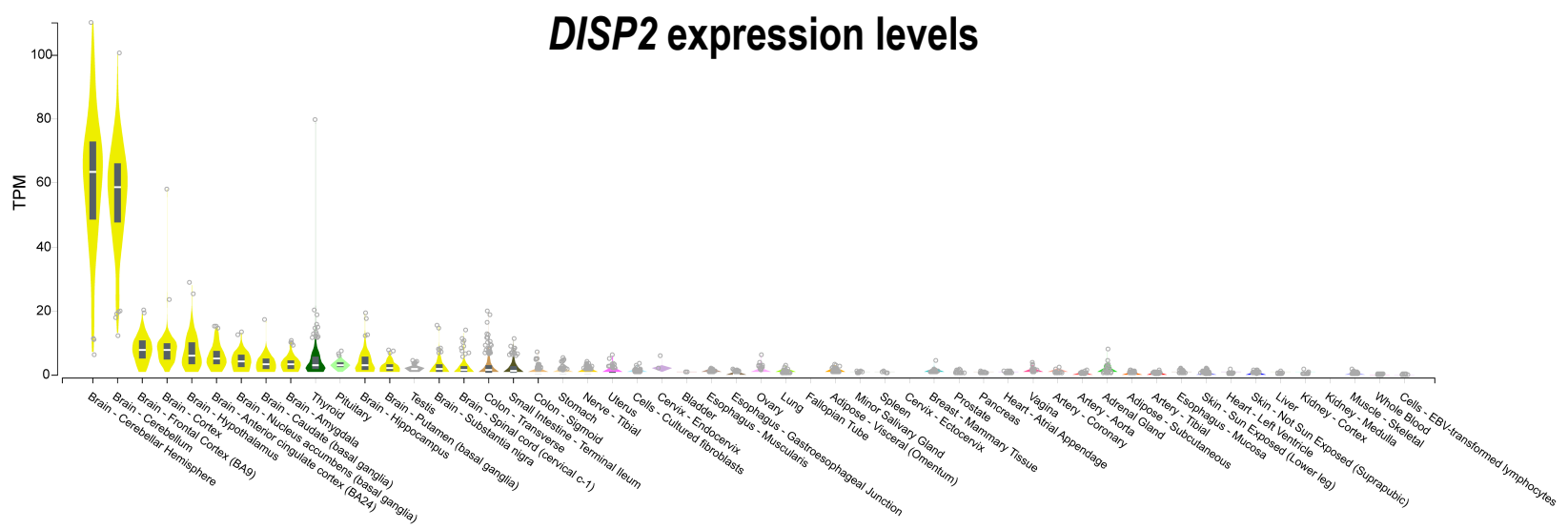


Supplementary Figure 2: Enhancer mapping and tissue expression of ZYG11A

A

Chr1:

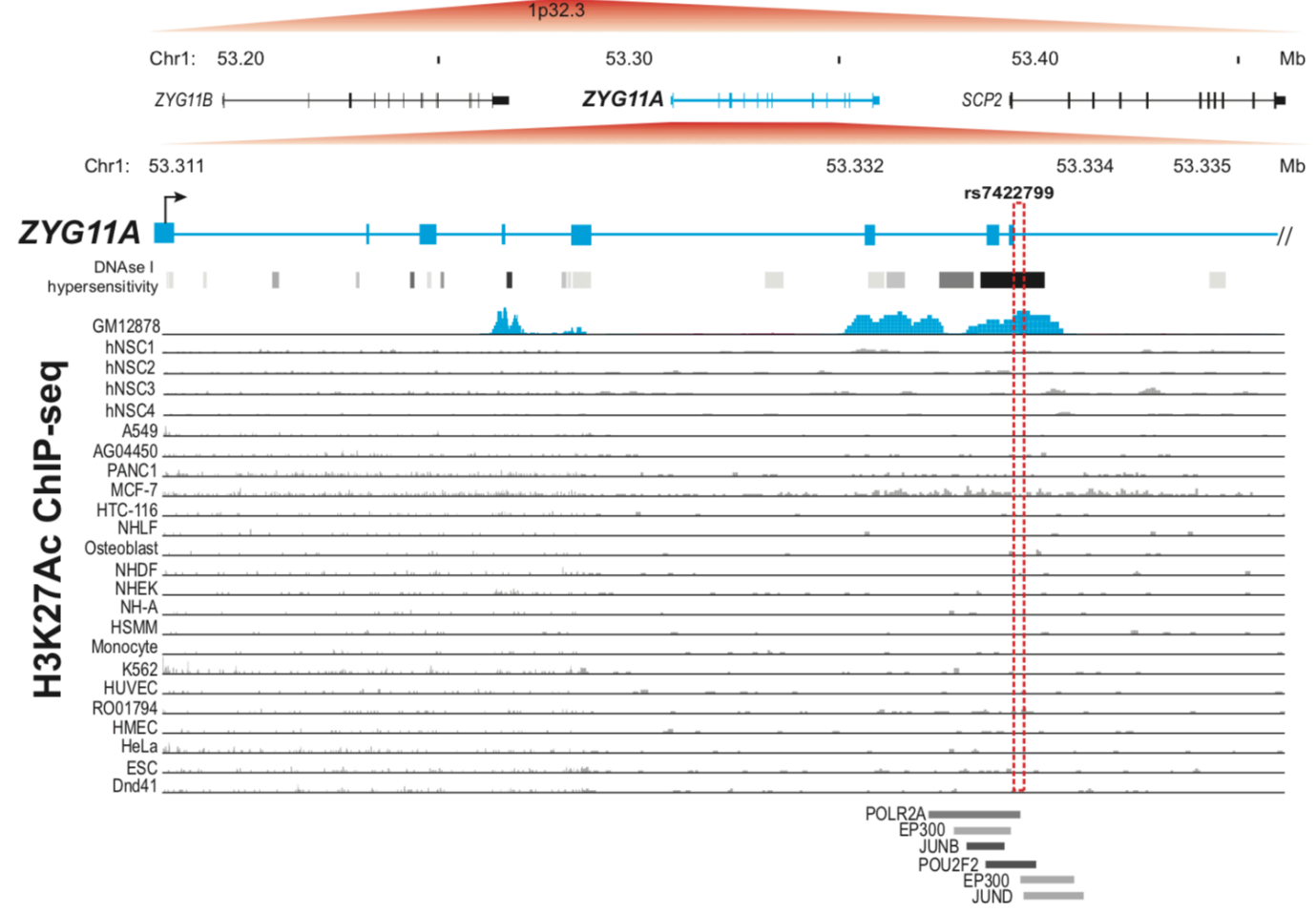

B

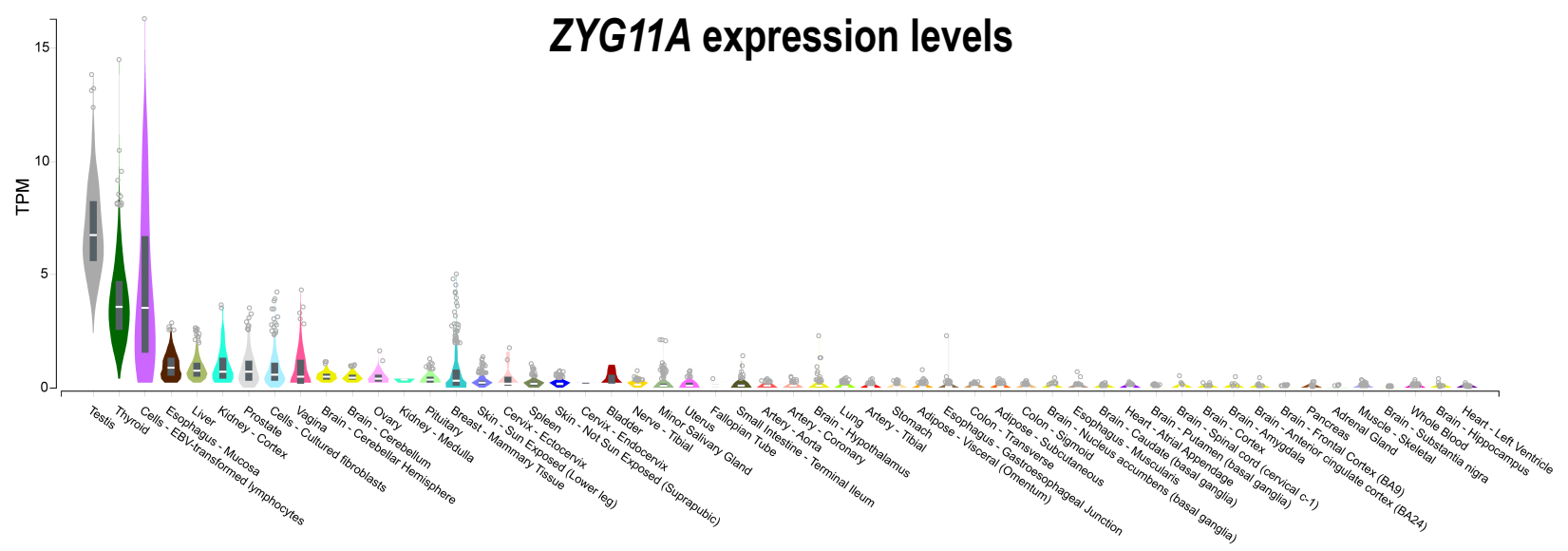

